\title{
Dark Sector Physics with Belle II
}

\section{Marcello Campajola*t}

Dipartimento di Fisica 'E. Pancini', Universitá di Napoli "Federico II" and INFN Napoli, Via Cinthia, 80126 Napoli, Italy

E-mail: marcello.campajola@na.infn.it

Belle II is a new generation of $B$-factory experiment, operating at the SuperKEKB energyasymmetric $e^{+} e^{-}$collider in Tsukuba, Japan. With a machine design luminosity of $8 \times 10^{35}$ $\mathrm{cm}^{-2} \mathrm{~m}^{-1}$ the Belle II experiment aims to record $50 \mathrm{ab}^{-1}$ of data within the next decade. Belle II offers the possibility to search for a large variety of Dark Sector particles with a mass below $10 \mathrm{GeV} / c^{2}$, being complementary to LHC and direct detection experiments. Belle II completed the first data taking runs for physics analysis and commissioning during 2018, and started full operation in early 2019. In this paper, we will review the status of the most promising dark sector searches to date at Belle II, with a focus on the discovery potential of the early data, and a preview of the first results.

XXIX International Symposium on Lepton Photon Interactions at High Energies - LeptonPhoton2019 August 5-10, 2019

Toronto, Canada

* Speaker.

${ }^{\dagger}$ on behalf of the Belle II collaboration. 


\section{Introduction}

Cosmological observations suggest that a considerable fraction of the mass of the Universe $(\sim 26 \%)$ is composed by the so-called Dark Matter (DM). Many efforts have been made to look for DM candidates that are charged under known Standards Model (SM) forces, like Weakly Interacting Massive Particles (WIMPs). However, the lack of experimental evidence, as well as independent theoretical motivation, triggered great interest into light-DM candidates neutral under SM and associated with new interactions. Such candidates with their interactions constitute the so-called "Dark Sector".

An interesting direction for the investigation of the dark sector is the one offered by the $B$ factories. In fact, because of the simple and clean initial state, $e^{+} e^{-} B$-factory experiments are particularly suitable for studying final states with missing energy and momentum, which is typical of events with DM production. Furthermore, by working at the so-called intensity frontier, they can reach an unparalleled sensitivity to the dark sectors within the $\mathrm{GeV}$ mass range, being complementary with respect to the energy frontier and direct search experiments.

In this paper we describe some of the main dark sector searches performed at the Belle II experiment, specifically the expected discovery potential for the searches of the dark photon $\left(A^{\prime}\right)$, axion-like particles (ALPs, $a$ ), and the preliminary results for the invisible decays of a $Z^{\prime}$ boson $\left(L_{\mu}-L_{\tau}\right.$ model $)$.

\section{SuperKEKB and Belle II}

Belle II is a full upgrade of the Belle experiment operating at the SuperKEKB $B$-factory, an $e^{+} e^{-}$energy-asymmetric collider that operates at the centre-of-mass $(\mathrm{CM})$ energy in the region of the $\Upsilon$ resonances. Belle II inherits the design of the Belle detector with major improvements in all of the subsystems. Higher beam current and more compact interaction region will allow SuperKEKB to provide a significant increase in the instantaneous luminosity (a factor of approximately 40) with respect to the precursor accelerator facility (KEKB). The detector and accelerator are described in detail here [1].

The final integrated luminosity goal of SuperKEKB and Belle II is $50 \mathrm{ab}^{-1}$, with a large portion of the data collected at the nominal collision energy $\sqrt{s}=10.58 \mathrm{GeV}$, which is the mass of the $\Upsilon(4 S)$ resonance. This higher integrated luminosity offer possibilities for a wide physics program, including dark sector physics and allow to obtain a great sensitivity to explore its parameter space. In addition to the luminosity increase, a major upgrade comes from the new single-photon and low multiplicity trigger system that allows much improved opportunity to search for dark sector physics.

The experiment completed the first data taking runs for physics analysis during 2018. During this period the experiment worked with a partially installed vertex detector at a reduced instantaneous luminosity for commissioning purpose, and approximately $0.5 \mathrm{fb}^{-1}$ of data were collected. In the early 2019 Belle II began the main operation with a near-complete detector and collected up to date approximately $6.5 \mathrm{fb}^{-1}$. 


\section{Search for invisible decay of $Z^{\prime}\left(L_{\mu}-L_{\tau}\right.$ model $)$}

A possible extension of SM involves a low mass $Z^{\prime}$ boson belonging to the Abelian symmetry indicated as $L_{\mu}-L_{\tau}$. Under this model, the $Z^{\prime}$ boson would couple only to the $2^{\text {nd }}$ and $3^{\text {rd }}$ generation of leptons with a new coupling constant $g^{\prime}$. The interaction Lagrangian term for such a model is given by:

$$
\sum_{\ell} \theta g^{\prime} \bar{\ell} \gamma^{\mu} Z_{\mu}^{\prime} \ell
$$

where the sum is extended to $\ell=\mu, \tau, v_{\mu}, v_{\tau}$ including the heavy leptons and their relative (lefthanded) neutrino species, with $\theta=-1$ if $\ell=\mu, v_{\mu}$ and $\theta=1$ if $\ell=\tau, v_{\tau}$.

The simplest way to look at such a $Z^{\prime}$ at an $e^{+} e^{-}$collider is with its production with a pair of muons $\left(e^{+} e^{-} \rightarrow \mu^{+} \mu^{-} Z^{\prime}\right)$, where $Z^{\prime}$ has been radiated by one of the two muons. Two different signatures are expected depending on whether it decays visibly, in $\mu$ or $\tau$ pairs, or invisibly, in SM neutrinos or DM if kinematically accessible. According to several models, such a boson can also be interpreted as the mediator of a new force that could create a bridge between SM particles and dark sector particles $[2,3]$. Thus, the invisible decay is particularly interesting since if the observed branching fraction is found to be in disagreement with that in SM neutrinos, it could be an indication of decay to DM.

A search for visible decays of the $Z^{\prime}$ boson to muons was performed by the BABAR experiment [4], while the invisible one is being performed for the first time at the Belle II experiment. The invisible decay final state consists of only two oppositely charged muons plus missing energy (see Fig. 1).

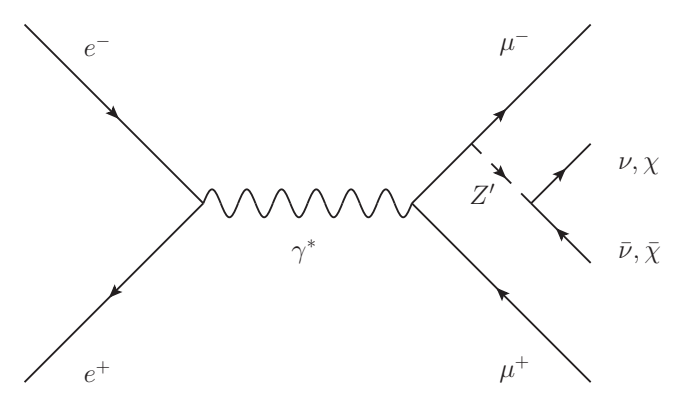

Figure 1: Feynman diagram for a $Z^{\prime}$ produced in $e^{+} e^{-} \rightarrow \mu^{+} \mu^{-}$events and decaying invisibly.

The experimental signature for the $Z^{\prime}$ production and its invisible decay would be a bump in the $Z^{\prime}$ candidate mass distribution which can be calculated by knowing the energy of $e^{+} e^{-}$collision and properties of the muons in the $\mathrm{CM}$ frame:

$$
m_{Z^{\prime}}^{2}=s+m_{\mu \mu}^{2}-2 \sqrt{s} E_{\mu \mu}^{C M}
$$

The main expected background sources are all the SM processes which give rise to final states with two tracks identified as muons plus missing energy. The relevant ones are $e^{+} e^{-} \rightarrow \mu^{+} \mu^{-}$, $e^{+} e^{-} \rightarrow \tau^{+} \tau^{-}$and $e^{+} e^{-} \rightarrow e^{+} e^{-} \mu^{+} \mu^{-}$.

By now a preliminary limit has been set for this process by using the data-set collected during the 2018 commissioning phase. Due to the low-multiplicity trigger configuration for this datataking campaign, only $0.276 \mathrm{fb}^{-1}$ were available. Fig. 2 shows the $90 \% \mathrm{CL}$ upper limits on the 
coupling constant $g^{\prime}$ as a function of the $Z^{\prime}$ candidate mass, where the solid line has been computed by assuming the $Z^{\prime}$ decays to SM neutrinos, while the dashed by assuming an enhancement to the $B F\left[Z^{\prime} \rightarrow\right.$ invisible $]=1$ as due to the presence of dark matter. No anomalies have been observed above $3 \sigma$ level.

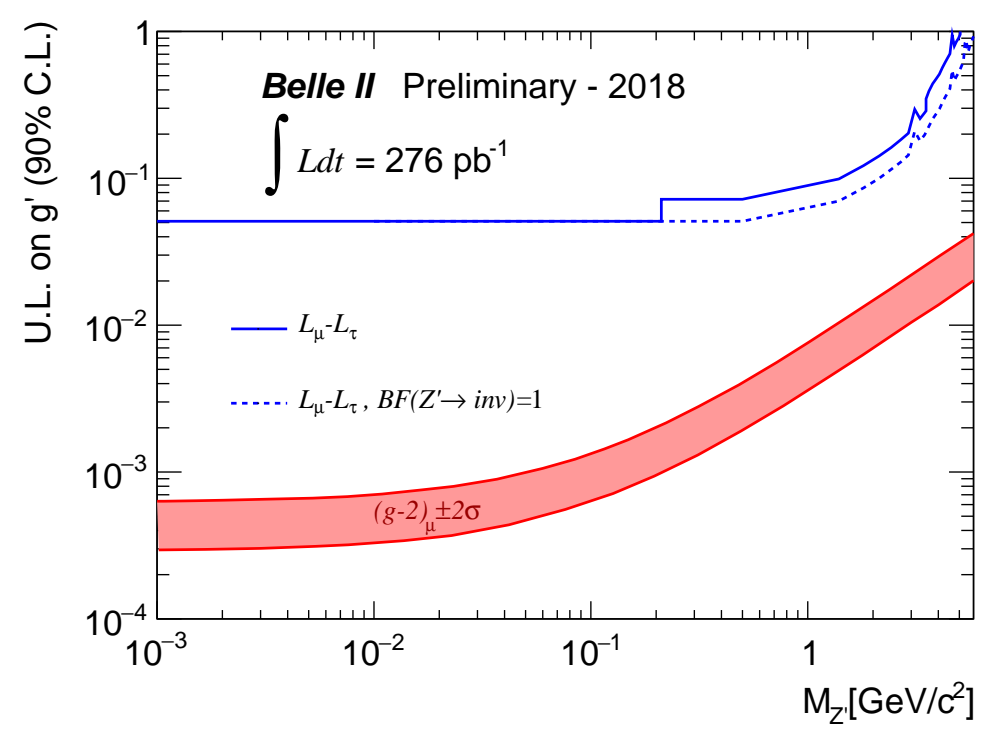

Figure 2: $90 \% \mathrm{CL}$ upper limits to $g^{\prime}$. The solid line assumes the $L_{\mu}-L_{\tau}$ model expected branching fraction for $Z^{\prime} \rightarrow$ invisible, while the dashed line assumes $B F\left[Z^{\prime} \rightarrow\right.$ invisible $]=1$. The red band shows the region that could explain the anomalous muon magnetic moment $(g-2)_{\mu} \pm 2 \sigma$.

\section{Search for visible decay of Axion-Like Particle}

Axion-like particles (ALPs) are often referred to as a possible extension of SM. They are hypothetical pseudo-scalar $\left(J^{P}=0^{-}\right)$particles that can couple to SM gauge bosons. Here we consider the case of coupling to SM photons, which is described by the following Lagrangian interaction term:

$$
-g_{a \gamma \gamma} / 4 a F_{\mu v} \tilde{F}^{\mu v}
$$

where $g_{a \gamma \gamma}$ is the coupling constant between the ALP and the SM photon. At an $e^{+} e^{-}$collider, there are two possible production mechanisms: ALP-strahlung and photon-photon fusion. Even if ALPs production via photon-photon fusion typically dominates over ALP-strahlung, this latter is experimentally more accessible as it results in a unique final state, with lower backgrounds. In this channel, three photons in the final state are expected (see Fig. 3), two of which with an invariant mass equivalent to that of the ALPs.

Depending on the properties of the ALPs model, i.e., the values of its mass $m_{a}$ and the coupling to the SM photon $g_{a \gamma \gamma}$, more experimental signatures are expected within the Belle II detector [5]. If the ALP is long-lived, it can escape from the detector volume before decaying. In this case, only a single photon is detected, which makes this analysis very similar to the one of the invisible Dark Photon search. The other possibility is that the ALP decays to two photons inside the detector volume, resulting in a three photon event. 


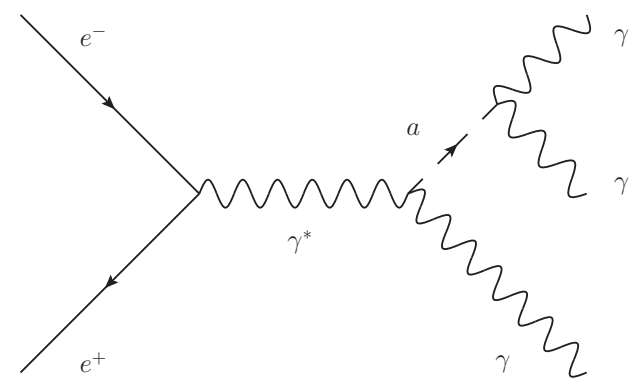

Figure 3: Feynman diagram of the ALP-strahlung production, with the subsequent decay $a \rightarrow \gamma \gamma$.

Here, we concentrate on the second case, for which the main background component is represented by the QED process $e^{+} e^{-} \rightarrow \gamma \gamma$ plus an additional radiated photon or beam background photon. The expected sensitivity (90\% CL) for the search of ALPs via the ALP-strahlung process with the data collected by Belle II during $2018\left(\sim 470 \mathrm{pb}^{-1}\right)$ is shown in Fig. 4 . Even with these first data collected, Belle II can improve the existing limits with a sensitivity to the $g_{a \gamma \gamma}$ down to $10^{-3}$.

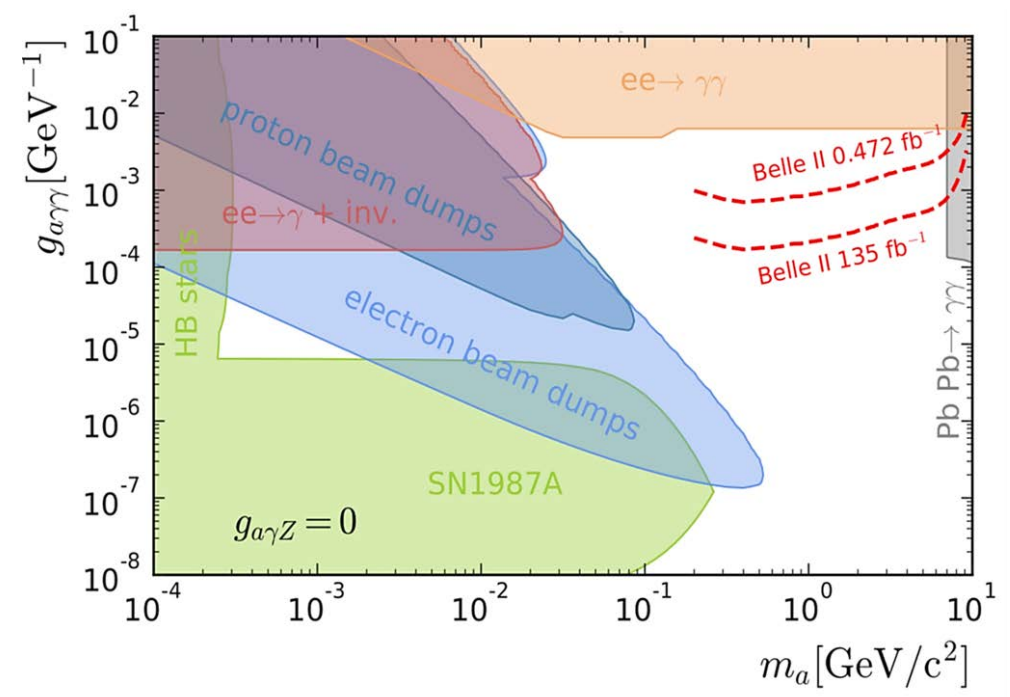

Figure 4: Belle II expected sensitivity to the ALPs search in early data-set.

\section{Search for invisible decay of Dark Photon}

Some of the minimal DM scenarios introduce a new mediator of a hypothetical dark force related to a $U(1)^{\prime}$ extension of the SM. Such a mediator is called dark photon $A^{\prime}$, and it is supposed to couple to SM through a kinetic mixing with the SM photon:

$$
\varepsilon A_{\mu}^{\prime} j_{E M}^{\mu}
$$

where $\varepsilon$ represent the strength mixing parameter between the electromagnetic current $j_{E M}^{\mu}$ and the dark photon $A_{\mu}^{\prime}$. 
In $e^{+} e^{-}$collisions the dark photon is searched in the reaction $e^{+} e^{-} \rightarrow A^{\prime} \gamma_{I S R}$ where $\gamma_{I S R}$ indicates an initial state radiation. Typically two decay channels for the dark photon can be investigated $A^{\prime} \rightarrow l^{+} l^{-}$and $A^{\prime} \rightarrow \chi \bar{\chi}$, which result in two distinct experimental signatures. In the case of the di-lepton decay, the signature is given by the presence of an energetic photon in the final state plus two oppositely charged tracks with an invariant mass equivalent to that of the dark photon. A search for the decay in a muon pair was done with the BaBar data-set of $514 \mathrm{fb}^{-1}$ [6]. On the other hand, if the $A^{\prime}$ is not the lightest particle of the dark sector, it is supposed to decay dominantly into light DM, i.e., $A^{\prime} \rightarrow \chi \bar{\chi}$ (see Fig. 5).

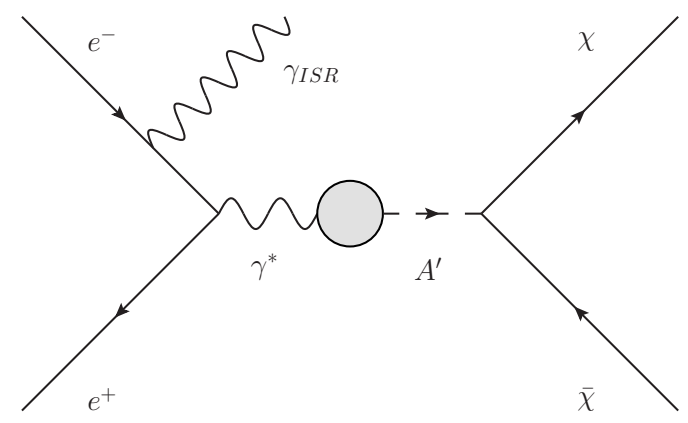

Figure 5: The Feynman diagram for a dark photon produced by kinematic mixing with a SM photon and decaying invisibly.

Since the probability of interaction of DM within the Belle II volume is negligible, the experimental signature of an on-shell dark photon production and decays to DM will be a mono-energetic ISR photon with energy:

$$
E_{\gamma_{I S R}}=\frac{s-m_{A^{\prime}}^{2}}{2 \sqrt{s}}
$$

This relation relates the ISR photon energy to the dark photon mass $m_{A^{\prime}}$ allowing one to precisely estimate it.

Searches for invisible decay of dark photon require the implementation of a single-photon trigger that was not available at the predecessor experiment Belle, but became available in Belle II. This kind of search is challenged by high background levels that could mimic the invisible dark photon decay signal. The main contribution to these backgrounds come from QED processes such as $e^{+} e^{-} \rightarrow e^{+} e^{-} \gamma$ where both the electron and the positron go outside the detector acceptance, and $e^{+} e^{-} \rightarrow \gamma \gamma$, with one photon undetected. The projected Belle II sensitivity to the kinetic mixing parameter strength $\varepsilon$ with an integrated luminosity of $20 \mathrm{fb}^{-1}$ is shown in Fig. 6 .

BaBar also performed this search with a datset of $53 \mathrm{fb}^{-1}$, due to the single-photon trigger availability [7]. It is interesting to note that, even with a smaller luminosity, Belle II should be able to set a much better limit than the analysis performed by BaBar. This is essentially due to the better hermiticity of the Belle II calorimeter compared to that of BaBar, due to the non-projective geometry of the crystals of the calorimeter which limits the loss of efficiency between adjacent crystal. Further improvement comes from the use of Belle II outer $K_{L}$ and Muon detector (KLM) as a veto for background events where a photon escapes detection through the calorimeter gaps. 


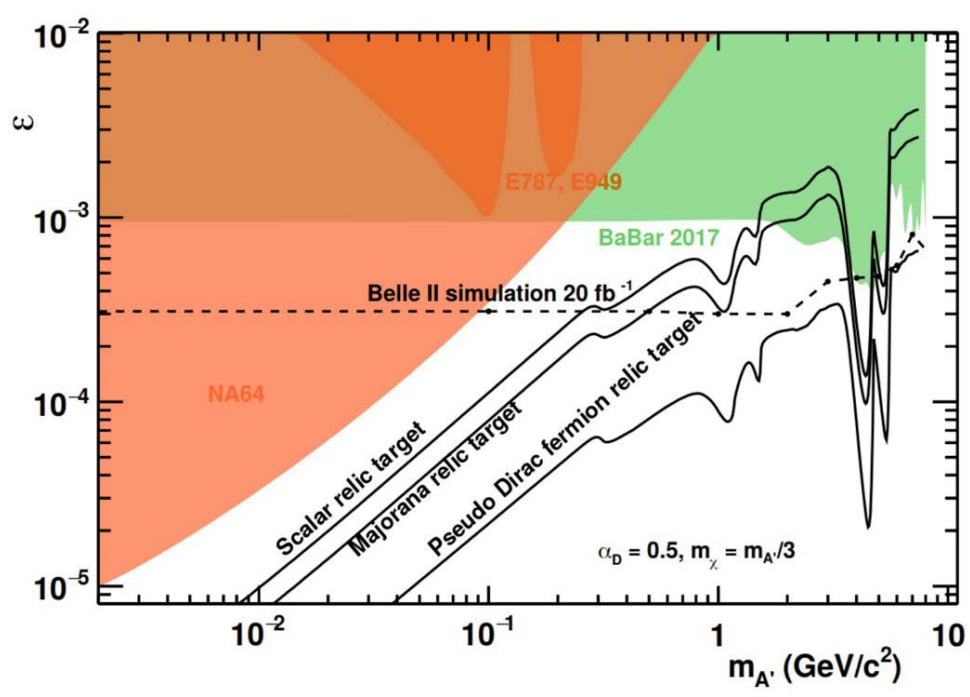

Figure 6: Belle II expected sensitivity to the kinetic mixing strength for invisible decays of the dark photon compared to other experiments [1].

\section{References}

[1] E. Kou et al. (Belle II Collaboration), The Belle II Physics Book, ArXiv: 1808.10567.

[2] B. Shuve and I. Yavin, Dark matter progenitor: Light vector boson decay into sterile neutrinos, Phys. Rev. D 89 (2014) 113004.

[3] W. Altmannshofer et al., Explaining dark matter and B decay anomalies with an model, J. High Energ. Phys. 12 (2016) 106.

[4] J.P. Lees et al. (BaBar Collaboration), Search for a muonic dark force at BaBar, Phys. Rev. D 94 (2016) 011102.

[5] M. Dolan, et al., Revised constraints and Belle II sensitivity for visible and invisible axion-like particles, J. High Energ. Phys. 12 (2017) 94.

[6] J.P. Lees et al. (BaBar Collaboration), Search for a dark photon in $e^{+} e^{-}$collisions at Babar, Phys. Rev. Lett. 113 (2014) 201801.

[7] J.P. Lees et al. (BaBar Collaboration), Search for Invisible Decays of a Dark Photon Produced in $e^{+} e^{-}$Collisions at BaBar, Phys. Rev. Lett. 119 (2017) 131804. 